\title{
Oportunidades de desarrollo de comunidades de investigación mediante la aplicación de la metáfora biológica
} Development Opportunities of Research Communities through the Application of Biologic Metaphors

\section{Iván Alonso Montoya ( \\ Colombia) \\ Universidad Nacional de Colombia \\ iamontoyar@unal.edu.co}

Profesor Asociado, Facultad de Minas, Universidad Nacional de Colombia.

\section{Luz Alexandra Montoya \\ (Colombia)}

Universidad Nacional de Colombia

lamontoyar@unal.edu.co

Profesora Asociada, Coordinadora Nacional del Concurso docente.

\section{Resumen}

La construcción y el desarrollo de comunidades de investigación en Colombia es un gran reto, en especial cuando el país solo invierte 0,37\% del PIB (Monsalve, 2011), a pesar de ello, los académicos han realizado importantes esfuerzos: el Ranking Iberoamericano sIR 2011 señala que en Colombia, la Universidad Nacional de Colombia ocupa el puesto 25 en Latinoamérica y el 56 en Iberoamérica (teniendo en cuenta a España y Portugal), seguido por la de Antioquia (Unimedios, 2011). Aunado a lo anterior, las redes de tecnologías avanzadas diseñadas para la investigación, aumentan sus convocatorias con el fin de que los investigadores trabajen

\section{Sandra Patricia Rojas \\ (Colombia) \\ Politécnico Grancolombiano}

srojasbe@poligran.edu.co

Directora Departamento de Investigación, Desarrollo e Innovación, Institución Universitaria Politécnico Grancolombiano.

\begin{abstract}
Construction and development of research communities in Colombia is a big challenge, especially when the country spends only $0.37 \%$ of gdp (Monsalve, 2011). In spite of this, scholars have made major efforts: the Latin American Ranking SIR 2011 notes that in Colombia, Universidad Nacional de Colombia is ranked 25th in Latin America and 56th in Iberoamerica (taking into account Spain and Portugal), followed by Universidad de Antioquia (Unimedios, 2011). In addition to the above, advanced technology networks designed to research, increase their callings so investigators can work in a network, prioritizing their inter-and trans-disciplin-
\end{abstract}


en red, priorizando así el trabajo inter y transdisciplinario (Dávila y otros, 2006), en dichos llamados, Colombia ha tenido una participación importante liderando e integrando comunidades.

Bajo este panorama, se propone la utilización de la metáfora biológica para la creación de oportunidades de desarrollo de comunidades investigativas. La aplicación de la metáfora biológica puede demostrar cómo las sociedades tienen mejor éxito adaptativo que los individuos trabajando aisladamente (Jaisson, 2000, 30). Los aportes de la sociobiología, como el estudio sistemático de los fundamentos biológicos del comportamiento social y de su evolución, (Thompson, 2003), en especial la inteligencia de enjambre, pueden ser aplicadas metafóricamente para la construcción de una comunidad de investigación con mayor oportunidad de desarrollo.

\section{Palabras clave}

Metáfora biológica, desarrollo de comunidades de investigación, Swarm Intelligence. ary work (Davila and others, 2006). In these callings, Colombia has had a significant participation by leading and integrating communities.

It is proposed, under this scenario, the use of the biological metaphor, for the creation of opportunities for the development of research communities. The application of the biological metaphor can demonstrate how societies have better adaptive success than individuals working alone (Jaisson, $2000,30)$. The contributions of sociobiology as the systematic study of the biological foundations of social behavior and its evolution (Thompson, 2003), especially swarm intelligence can be applied metaphorically to the construction of a research community with greater opportunity for development.

\section{Keywords}

Biological Metaphor, Research Community Development, Swarm Intelligence. 


\section{Introducción}

Las metáforas aparecen en las organizaciones con el fin de hacer familiar lo que no es familiar, como un mecanismo que además de poner límites en el cuerpo del conocimiento sobre las organizaciones (Cornelissen et al. 2005), permite pensar la organización de nuevas formas (Cox \& Minahan 2006); es así como, las metáforas se convierten en los modelos mentales que los investigadores organizacionales tienen, de manera que el desarrollo de sus teorías llevan a los investigadores a comprender a las organizaciones de forma distinta y parcial (Cadavid, 2010, Montoya, 2010).

En estos ámbitos, la metáfora permite el surgimiento de una nueva teoría científica o filosófica, la cual suele tener en su base, o generar como resultado, una nueva metáfora creativa y una red de metáforas subsidiarias de ella con, al menos, tres consecuencias importantes:

- Proponer un nuevo modelo o un nuevo marco de referencia para conocer la realidad.

- Crear una red de metáforas subsidiarias, lo que permite generar un número indefinido de aseveraciones, sobre esa realidad, congruentes con la metáfora básica.

- Entrar en colisión y sustituir, si tiene éxito, a las teorías rivales anteriores y contemporáneas, cuyas redes de metáforas se muestren incompatibles con la nueva (Vattimo, 2002).

Por su parte, la construcción y el desarrollo de redes de investigación precisan de un modelo conceptual que les permita aunar esfuerzos para alcanzar metas que permitan una verdadera cohesión, apoyo en los mismos y resultados que redunden en el avance del conocimiento y en el desarrollo social.

\section{Aplicación de la metáfora biológica}

Dentro de las diferentes metáforas biológicas conocidas para la aplicación de temas económicos y en especial sociales (Montoya, 2010), se encuentra el concepto de "Cooperón social", aplicado desde los modelos sociobiológicos (Wilson, 1995). Esta estructura organizacional de los seres vivos, destaca el cómo la asociación de individuos multicelulares (integración entre individualidades complementarias y mutualistas) de la misma especie, en comunidades estables y organizadas: las sociedades, con divisiones de las funciones entre sus miembros (Jaisson, 2000, 30). Su división social se caracteriza por una formalización llevada al extremo, lo que define el nivel de socialidad más avanzado desde el punto de vista de la evolución, demostrando que comparadas con los individuos solitarios, las sociedades aportan una ventaja considerable al garantizar la rentabilidad del trabajo de cada uno de sus miembros, aun cuando este trabajo quede inconcluso por la muerte de un individuo, entonces es recuperado y continuado por sus compańeros, lo que aumenta la rentabilidad selectiva del grupo (Wilson, 1995, Alcock, 2001).

El "Cooperón social" surge como un hecho de la evolución, una forma de vida que organiza la existencia de los individuos, de tal manera, que este modo de vida presenta ventajas adaptativas que justifican su éxito. Un ejemplo de ello es la hormiga roja japonesa fórmica yessensis, que vive junto al mar de Japón, con menos de un centímetro de longitud ha constituido una federación de 45.000 colonias que ocupan 270 hectáreas y reúnen 
más de 300 millones de obreras y 1,1 millones de reinas (Wilson, 1995, Alcock, 2001, Montoya, 2010).

Este éxito adaptativo de las sociedades de insectos demuestra que las estructuras cooperativas son más eficaces que los individuos aislados. En la competencia impuesta por la selección natural, se es más fuerte cuando se asocia uno para constituir no como una simple colección de individuos, sino como una especie de superindividuo, más apto para sobrevivir y garantizar con ello la supervivencia de cada uno de sus miembros (Alcock, 2001).

Dentro de las estrategias que tienen los grupos sociales biológicos, puede encontrarse el concepto de "Swarm Intelligence", el cual se señala como el surgimiento de un comportamiento colectivo, y que se traduce como una inteligencia de enjambre o cardumen.

El "Swarm Intelligence" señala que los insectos sociales tienen éxito en la ecoesfera- por lo menos por tres características:

- Flexibilidad, (la colonia puede adaptarse a los cambios del ambiente): el grupo puede adaptarse rápidamente a los cambios del ambiente.

- Robustez, (cuando uno de los miembros muere y los otros lo reemplazan rápidamente): cada uno de los individuos puede realizar su tarea, todos son necesarios pero nadie es indispensable, por ejemplo con una adecuada gestión de recursos humanos se puede tener polivalencia, alta rotación y socializar el know how individual.

- Autoorganización: el grupo necesita relativo poco control y supervisión, las actividades no requieren de un control central o de supervisión local (Zimmer, 2007, 108), (Pereira, 2008).

En la aplicación práctica, además se ha encontrado que un sistema de inteligencia de enjambre típico tiene las siguientes propiedades:

- Se compone de muchas personas; los individuos son relativamente homogéneos (es decir, son todos idénticos o pertenecen a unas tipologías).

- Las interacciones entre los individuos se basan en simples reglas de comportamiento, que explotan solo información local que los individuos intercambian directamente o a través del entorno (estigmergia).

- Es un sistema que se autoorganiza. La propiedad característica de un sistema de inteligencia de enjambre, es su capacidad para actuar de forma coordinada sin la presencia de un coordinador o de un controlador externo.

- Tiene "Escalabilidad", es decir, que permite que un sistema pueda mantener su función mientras aumenta su tamaño sin necesidad de redefinir la forma en que interactúan sus partes.

- Las personas pueden realizar diferentes acciones en diferentes lugares al mismo tiempo, denominada "Acción paralela" (Bonabeau, 1999).

\section{Aplicaciones de la metáfora biológica en las redes académicas}

Para la Universidad Nacional de Colombia, en sus dos estudios sobre planta docente, realizar la analogía puede 
considerarse en extremo interesante. Señala cómo la colonia de hormigas, como metáfora de la sociedad o la organización, revela una profunda concepción de igualdad y equidad en la labor de todas y cada una de ellas. Así mismo, parecería estar presente una concepción del mundo donde todos tienen trabajos iguales y cargas iguales. Detrás de esta idea, estaría implícita la noción de que las hormigas actúan como una entidad única, que trabajan colectivamente en apoyo a la colonia.

Es así, como se compara el comportamiento de dichas hormigas, frente al comportamiento por ejemplo de las avispas: "dentro de la múltiple variedad de avispas que se encuentran, hay unas solitarias y otras sociales. Las especies solitarias son parásitas, más específicamente parasitoides. Otras tantas especies son predadoras; por lo cual, cada vez es más común el uso de avispas como pesticida natural. Hay otras especies de avispas que se alimentan de polen, néctar, frutas, hojas o hasta carroña. Incluso hay avispas omnívoras" (Vicerrectoría académica, Universidad Nacional de Colombia, 2010, 2).

La metáfora de las hormigas muestra una organización donde todos contribuyen por igual a la cohesión del cuerpo social. En cierto sentido, bajo esta metáfora, la organización prevalece y el individuo desaparece. Por otro lado, a pesar de las diferencias, la metáfora de las avispas revela identidad. Las avispas son un grupo bastante heterogéneo y diverso, donde cualquier comparación entre especies resulta inadecuada, pero, a pesar de sus diferencias, todas ellas siguen siendo avispas; ¿qué las diferencia y qué tienen en común? es la pregunta que debe guiar un análisis sobre ellas y su forma de organización (Vicerrectoría Académica, Universidad Nacional de Colombia, 2010-2011).
Por su parte, Redclara que interconecta físicamente a las redes académicas avanzadas nacionales de Latinoamérica y a éstas con las redes de Europa (GÉANT2), Estados Unidos (Internet2), Asia (APAN) y el resto del mundo, ha otorgado a los científicos, académicos e investigadores, una infraestructura provista para el trabajo colaborativo, la topología de dicha red se configura de la siguiente manera:

Figura 1. Topología de Redclara

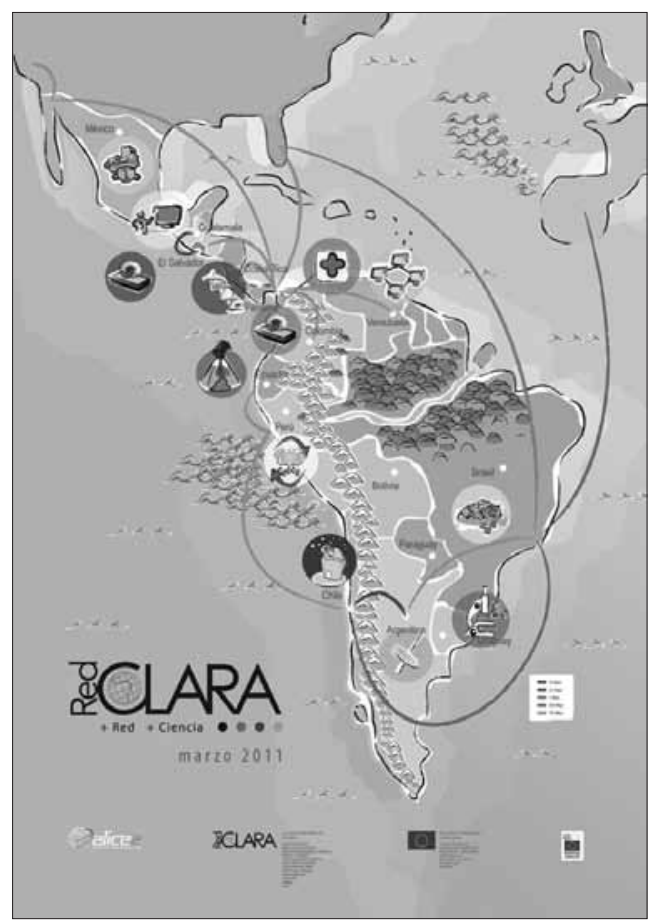

Fuente: http://redclara.net/index.php?option=com_co ntent\&view=article\&id $=51 \&$ ltemid $=422 \&$ lang $=$ es $^{\prime}$

Los servicios que ofrece a la comunidad latinoamericana de investigadores son:

- IPv4.

1 Recuperado 22-08-2011. 
- Multicast.

- IPv6.

- Multicast IPv6.

- Disponibilidad de ancho de banda (QoS).

- Mediciones.

- Servicios específicos para proyectos: mallas computacionales (Grids).

Sin embargo, existen algunas problemáticas como que:

- La concentración de la masa crítica en un solo país, Brasil, que reúne casi al $50 \%$ de los investigadores de la región, luego se agregan Argentina y México. Si se añaden a la lista a Chile, Colombia y Venezuela, se configura un cuadro que involucra a más del $80 \%$ de la masa crítica de los usuarios naturales de las redes avanzadas (Clara; 2010, p. 11).

Por otro lado, estas redes académicas de alta velocidad en contextos como el europeo o norteamericano, no privilegian áreas del conocimiento en particular sino que promueven el uso y conectividad para todas ellas. Por su parte, en Latinoamérica fue tan solo hasta 2010 que se realizó una iniciativa que permitiera que dicha conexión física trascendiera de ello para llegar a ser una red de colaboración, el mecanismo para lograrlo fue de una convocatoria para conformar comunidades de investigación, que contó con una segunda versión en 2011 y cuyos resultados comparados se observan en la siguiente tabla:
Tabla 1. Resumen de convocatorias para

conformación de Comunidades de Redclara

\begin{tabular}{|c|c|c|c|c|}
\hline Comunidad & País & Temática & 2010 & 2011 \\
\hline COLABORA & Colombia & Educación & ok & ok \\
\hline LACLO & Ecuador & Educación & ok & ok \\
\hline LACXER & Colombia & Ingeniería & ok & $!$ \\
\hline LAGO & Venezuela & Ingeniería & ok & ok \\
\hline Mapa D2 & Brasil & Artes & ok & $!$ \\
\hline MAYA & Perú & Agricultura & ok & $!$ \\
\hline MCI Sur & Uruguay & Sectores Específicos & ok & $!$ \\
\hline RELANS & Costa Rica & Nanotecnología & ok & $!$ \\
\hline URDIMBRE & Colombia & Educación & ok & $!$ \\
\hline ACHALAI & Guatemala & Salud & $\mathrm{X}$ & ok \\
\hline ARCU-RED & Colombia & Artes & $\mathrm{X}$ & ok \\
\hline CLARISE & México & Educación & $\mathrm{X}$ & ok \\
\hline CLIC & Colombia & Educación & $\mathrm{X}$ & ok \\
\hline COMHPC-GRID & Colombia & TIC-Mallas & $\mathrm{X}$ & ok \\
\hline FLU-CAP & Guatemala & Salud & $\mathrm{X}$ & ok \\
\hline IPOL-LA & Uruguay & Salud & $\mathrm{X}$ & ok \\
\hline LATIN DE & Ecuador & Desastres naturales & $\mathrm{X}$ & ok \\
\hline MAPA D2 & Brasil & Patrimonio cultural & $\mathrm{X}$ & ok \\
\hline
\end{tabular}

ok se formaliza en CLARA o Permanece en ella

$\mathrm{x}$ no folmal en CLARA

sin información de permanencia

Fuente: la investigación.

De la anterior información, se evidencia que tan solo tres comunidades han permanecido formalmente en CLARA, cabe destacar que en 2011, la red inauguró un portal de servicios que permite a los investigadores acceder a herramientas como red social, videoconferencia de escritorio, Wikis, alertas de fondos de investigación, búsqueda de pares, integración a comunidades, conformación de nuevas comunidades, entre otros.

En el siguiente apartado se exponen algunas alternativas de gestión que contribuirán a retroalimentar las aplicaciones aquí expuestas.

\section{Discusión}

Muchos sistemas biológicos, sociales o de comunicación, se pueden describir adecuadamente a través de redes complejas cuyos nodos representan individuos $u$ 
organizaciones, y los enlaces simbolizan las interacciones entre ellos. Una clase importante de redes son aquellas que cumplen con las reglas de un "mundo pequeño", cuya topología exhibe dos rasgos esenciales: todo nodo está fuertemente conectado con muchos de sus vecinos pero débilmente con algunos pocos elementos alejados (fenómeno conocido como apińamiento, agrupamiento o clustering) y todo nodo puede conectar a cualquier otro con solo unos cuantos saltos (en otras palabras, existe una pequeña "distancia" entre ellos). Esto implica dos cosas: que la información se transfiere muy rápidamente entre dos elementos cualquiera, y que existe un pequeño número de nodos claves por donde circula un gran porcentaje del tráfico total (Moriello, 2010).

Aparentemente, estas redes siguen el mismo patrón de auto-organización de los sistemas complejos biológicos: los nuevos nodos agregados tienden a formar conexiones con aquellos que ya están bien conectados (las partes conductoras mencionadas en el apartado anterior). Por ejemplo, los nuevos artículos científicos ci$\tan$ a otros ya bien establecidos y las nuevas páginas en Internet se conectan a los buscadores más conocidos. De allí, que los hubs también parecen ser los responsables de mantener la cohesión de este tipo de redes e, incluso, de permitirle evolucionar, ya que pequeñas perturbaciones en ellos pueden ocasionar cambios en el funcionamiento de la red. Así mismo, algunos investigadores especulan ya que, en algunos casos, tienden a aclarar que los sistemas naturales evolucionan hacia redes de mundo pequeño, porque tienen una elevada tolerancia a las fallas (la conexión de cualquier pareja de nodos puede establecerse a través de varios caminos alternativos), y hacia redes independientes de la escala, porque utiliza más eficientemente los recursos que las redes aleatorias (resuelve adecuadamente el conflicto entre las necesidades de bajo costo y alto rendimiento).

Aun cuando su funcionamiento puede ser muy diferente entre una red y otra, el hecho de que compartan la misma topología permitiría estudiar las más complejas a partir de las más simples. Así, por ejemplo, si las redes neuronal y genética pertenecieran a la misma categoría genérica, los científicos podrían aprender mucho más sobre el sistema nervioso escudriñando el sistema genético, el cual es relativamente más sencillo (Moriello, 2010, 10).

Por ejemplo, una hormiga considerada aisladamente es una criatura tonta, únicamente capaz de ejecutar -aunque de forma fiel y obstinada - un pequeño conjunto de rutinas innatas, pero condicionada por el entorno circundante. No obstante, tomadas en grupo, son capaces de erigir sociedades complejas con sofisticadas actividades como la agricultura, ganadería, arquitectura, ingeniería e, incluso, prácticas de esclavitud. De esta forma, podría considerarse al hormiguero como un macroorganismo, que presenta un comportamiento global inteligente. Es decir, nadie planifica, nadie ordena ni controla, pero surge un comportamiento colectivo -quizás instintivo- o una necesidad que las "obliga" a trabajar juntas persiguiendo un fin común (Moriello, 2010).

El algoritmo de búsqueda funciona de la siguiente manera: cada hormiga virtual -de un conjunto enormemente grande de ellas- sale en busca de "alimento" alrededor de su "hormiguero" (el punto de partida), de una manera más o menos al azar. Entonces, cada insecto "marca" el camino realizado con una "feromona" (la cual guarda cierta relación con la "longitud" o distancia recorrida) que otras pueden seguir. Dado que la feromona se "evapora" 
con el tiempo, las "buenas" rutas (las más cortas) se hacen más atractivas que las "malas" para el resto de las hormigas, con lo cual se intensifica cada vez más el rastro de feromonas en esa ruta. Al final del proceso, lo habitual es que se seleccione el rastro más fuerte, que justamente es la ruta más corta entre el punto de partida y el punto de llegada. Lo interesante de esta técnica es que las hormigas pueden adaptarse al entorno: dado que éste es dinámico, es posible que surjan determinadas complicaciones, como el bloqueo o la congestión en las rutas (Moriello, 2010).

Desde esta perspectiva, se puede afirmar que un rasgo central de las redes es su autonomía y consecuentemente su capacidad de autorregulación. Consideradas como sistemas complejos de relaciones, que resultan de procesos simultáneos de diferenciación e interdependencia, entre individuos, grupos, instituciones $\mathrm{o}$ subsistemas diversos; las redes de conocimiento son autónomas en un doble sentido. Por una parte, cada uno de sus componentes es autónomo y permanece como tal aun cuando la interacción y la colaboración se intensifiquen. Por otra parte, el conjunto de la red es autónoma, en el sentido de que no está sujeta a una entidad superior que regule sus acciones. Esta doble autonomía significa que no hay reglas preestablecidas -ya sea que provengan de fuera o dentro de la red- que determinen los derechos y obligaciones de los miembros y los procedimientos que sus interacciones deben seguir. El hecho de que ningún miembro tiene una total autoridad y todos tengan una cierta autonomía, se manifiesta en una dispersión dinámica de la autoridad (León, Velazco, 2005, 1).

La confianza basada en el prestigio depende sobre todo de las percepciones acerca de las capacidades y las competencias de los participantes (que pueden ser individuos u organizaciones). La confianza calculada o estratégica surge de estimaciones de costos y beneficios; por lo tanto, depende de la expectativa de ganancias mutuas que puede generar la relación. Un problema relacionado con este tipo de confianza es que, dada la naturaleza compleja de las redes y la heterogeneidad de sus participantes, la carencia de criterios compartidos para medir las pérdidas y ganancias, incrementa los costos de la negociación y hace más necesaria la deliberación (León, Velazco, 2005, 2).

Finalmente, la confianza personal o normativa depende de normas, creencias y valores compartidos; se basa en la solidaridad social, más que en las ganancias esperadas de la interacción. Por otra parte, la confianza personal es particularmente relevante para el flujo de conocimiento tácito, mientras que la técnica facilita los flujos de conocimiento codificado o formal (Albornoz \& Alfaraz, 2006, p. 22).

\section{Implicaciones para futuras investigaciones}

Futuras investigaciones deberían revisar las comunidades de investigación en Latinoamérica y revisar los siguientes aspectos:

- Los modelos de gestión de conocimiento generados al interior de las comunidades.

- Los productos y resultados de investigación de las comunidades.

- El aporte de dichos productos al desarrollo de la región en términos económicos y sociales. 


\section{Bibliografía}

I. Bonabeau, E. Dorigo M, and Theraulaz G. (1999). Swarm Intelligence: From Natural to Artificial System. Oxford University Press, New York.

2. Cadavid, Lorena (2010). Propuesta para la medición del ajuste entre las metáforas organizacionales y las herramientas de modelado. Facultad de Minas, Universidad Nacional de Colombia, Medellín.

3. Dávila, J, Nuñez, L, Sandía, B y Torréns, R. (2006). Los repositorios institucionales y la preservación del patrimonio intelectual académico. En: Revista Interciencia, volumen 31, No. 1.

4. Garzón, Díaz, R. (2005). Programa sobre operaciones colectivas entre empresas, Convenio Bid-Atn/Me 7856 Caracas.

5. www.iberpymeonline.org/Venezuela130705/Ricardogarzon.Pdf

6. Jaisson P. (2000). La hormiga y el sociobiólogo. Fondo de Cultura Económica, México.

7. León, Velazco (2005). Redes de conocimiento: principios de coordinación y mecanismos de integración, Seminario "Redes de conocimiento como nueva forma de creación colaborativa: su construcción, dinámica y gestión.

8. Monsalve A. (2011). Invertir en ciencia, tecnología e innovación o desaparecer. En: Revista Cambio, viernes 27 de mayo de 2011. Disponible en:

9. http://www.cambio.com.co/paiscambio/752/ARTICULO-WEB-NOTA INTERIOR CAMBIO-3836416.html

Io.Montoya, A, Montoya, I, Rojas, S. (2011). Enfoques biológicos de la gestión: desde la competencia hasta la cooperación, xv Congreso Internacional de Investigación en Ciencias Administrativas Boca del Río, Veracruz, 17 al 20 de mayo de 2011.

I I.Moore James. 2005. Business Ecosystems and the View from the Firm. The Antitrust Bulletin/Fal.

I 2. Moreno, Alejandro, Lara, Arturo. (2007). Coevolución de las capacidades metrológicas del Centro Nacional de Metrología y del Sistema Petrológico Nacional en relación a la demanda de los usuarios del sector automotriz en México, en el periodo 1994-2005. Universidad Autónoma Metropolitana, México D. F.

I 3. Morgan, Gareth. (1991). Imágenes de la organización. Alfaomega, México.

I 4. Moriello, Sergio. (2010). Sistemas complejos, caos y vida artificial. Pensamiento complejo. Disponible en: pensamientocomplejo.com.ar

I 5. Nooteboom, Bart. (2003). Organizational Learning and Multinational Strategy Rotterdam School of Management, Erasmus University Rotterdam.

i6. Núñez Manuel y Moyano. (2002). Nuevas medidas de tamaño en ecología poblacional. Departamento de Economía de la Empresa. Madrid.

I7.Pereira, J. Chica, M. Cordón, O. Damas, S. Bautista, J. (2008), Incorporating Preferences to a Multi-Objective ant Colony Algorithm for Time y Space Assembly Line Balancing, Lecture Notes in Computer Science-Ant Colony Opmization y Swarm Intelligence, Vol. 5217/2008, Springer, 331-338.

I 8. ricyt-cyted, Buenos Aires, 24 y 25 de noviembre de 2005 disponible en: http://www.hacienda.go.cr/centro/datos/Articulo/Redes\%20de\%20conocimiento.pdf

I9. Riolo, Rick L., Michael D. Cohen and Robert Axelrod, "Evolution of 
Cooperation Without Reciprocity". Nature, 414, 441-443, (22 Nov. 2001).

20.Scherer, F.M. y Ross, D. (1990). Industrial Market Structure and Economic Performance, Houghton Mifflin Company, Boston, Massachusetts.

2I.Shapiro, Carl and Hal R. Varian. (1998). Information Rules: A Strategic Guide to the Network Economy. The Journal of Technology Transfervolume 25, Number 2. Junio de 2000.

22.Sheldrake, Rupert. (1990). The Presence of the Past. Londres.

23.Sheldrake, Rupert. (1990). Una nueva ciencia de la vida. La hipótesis de causación formativa. Editorial Kairos, Barcelona.

24. Taboada, Eunice. (2003). "La cooperación tecnológica inter firma desde la teoría económica”, Avances de tesis, doctorado en Ciencias Económicas, uam.

25. Thompson, J. (2003). El proceso evolutivo, Fondo de cultura económica, México.

26. Thompson, John. (2003). El proceso evolutivo, Fondo de Cultura Económica, México.

27. Unimedios. (2011). Agencia de Noticias un. un, líder en el país en publicaciones científicas, May. 20 de 201. Disponible en : http://www.agenciadenoticias. unal.edu.co/Nc/Detalle/Article/Un-lider-en-el-pais-en-publicaciones-cientificas.html

28.Vattimo, (2002). "Some Theses on Euphemisms and Dysphemisms". En:
Mind, Language and Metaphor: Euroconference on Consciousness and the Imagination. Celebrado en Kerkrade (Holanda) del 20 al 24 de abril de 2002. 29. Vicerrectoría Académica. (2011). Arañas y telarañas, estudio de la planta docente de la Universidad Nacional de Colombia, 2010. Universidad Nacional de Colombia, Bogotá.

30. Vicerrectoría Académica. (2010). ¿Hormigas o avispas? Estudio de la planta docente de la Universidad Nacional de Colombia 2010. Universidad Nacional de Colombia, Bogotá.

3 I. Weissenberger-Eibl, M., Koch, D.J. Collaboration, Network Formation and Co-Opetition in International Service Businesses, 2005. University Kassel.

32. Wilson, Edward. (1995). Science and Ideology. Academy Questions. Summer.

33. Wilson, Edward. (2002). Sociobiology, the new Synthesis. 25th Anniversary Ed. Harvard Collage.

34.Zimmer, Carl. Distintas especies comparten un instinto de grupo. En: The New Cork Times, 2 de diciembre de 2007. El Tiempo, Bogotá. 